\title{
BMJ Open What hinders congenital ectopia lentis patients' follow-up visits? A qualitative study
}

\author{
Jianqiang Lin (1) , ${ }^{1}$ Ni Gong, ${ }^{2}$ Qianzhong Cao, ${ }^{1}$ Yijing Zhou, ${ }^{1}$ Yitingxue Cai, ${ }^{3}$ \\ Guangming Jin, ${ }^{1}$ Charlotte Aimee Young, ${ }^{4}$ Jing Yang, ${ }^{1}$ Yiyao Wang, ${ }^{1}$ \\ Danying Zheng ${ }^{1}$
}

To cite: Lin J, Gong N, Cao $Q$, et al. What hinders congenital ectopia lentis patients' follow-up visits? A qualitative study. BMJ Open 2020;10:e030434. doi:10.1136/ bmjopen-2019-030434

- Prepublication history for this paper is available online. To view these files, please visit the journal online (http://dx.doi org/10.1136/bmjopen-2019030434).

$\mathrm{JL}$ and NG contributed equally.

Received 14 March 2019 Revised 06 January 2020 Accepted 11 February 2020

Check for updates

(c) Author(s) (or their employer(s)) 2020. Re-use permitted under CC BY-NC. No commercial re-use. See rights and permissions. Published by BMJ.

${ }^{1}$ State Key Laboratory of Ophthalmology, Zhongshan Ophthalmic Center, Sun YatSen University, Guangzhou, Guangdong, China

${ }^{2}$ School of Nursing, Sun Yat-Sen University, Guangzhou, China

${ }^{3}$ School of Sociology and Anthropology, Sun Yat-Sen University, Guangzhou, Guangdong, China ${ }^{4}$ Department of Ophthalmology, University of California, San Francisco, California, USA

Correspondence to Dr Danying Zheng; zhengdyy@163.com

\section{ABSTRACT}

Objectives The aim of our study is to give insight into congenital ectopia lentis (CEL) patients' care-seeking behaviour and explore the factors affecting their follow-up visits.

Design Cross-sectional study; in-depth and face-to-face semistructured interview.

Setting A large-scale ophthalmology hospital in China. Participants 35 patients with CEL and their parents from May 2017 to August 2017.

Main outcome measures Themes and categories. The interviews were audio-recorded, transcribed verbatim, coded and analysed using grounded theory. Data collection was closed when new themes did not emerge in subsequent dialogues.

Results The factors affecting the timely visits included insufficient awareness of CEL, shame on hereditary disease, lack of effective doctor-patient communication, lack of reliable information online and daily stressors.

Conclusion Continuing medical education of severe and rare disease, reforming the pattern of medical education, constructing an interactive platform of the disease on the internet and improving healthcare policy are effective ways to improve the diagnosis and treatment status of CEL in China.

\section{INTRODUCTION}

Congenital ectopia lentis (CEL) is a disease caused by congenital dysplasia of the zonule, which leads to the crystalline lens deviating from its normal position. The incidence rate of CEL is approximately $6.4 / 10$ million, ${ }^{1}$ and most of these cases are genetic diseases. CEL may occur alone or combined with abnormalities in other systems such as the skeletal system and cardiovascular system, can result in the onset of diseases such as Marfan syndrome and homocystinuria. ${ }^{2}$ The lesions of the cardiovascular system include mitral valve prolapse, aortic dilation, aortic aneurysm and aortic dissection, which may seriously endanger a patient's life. ${ }^{3}$ The dislocation of the lens is usually the initial symptom and occurs before the age of $10 .^{4}$ Because visual impairment may affect a patient's daily
Strengths and limitations of this study

- Ophthalmological symptoms are the initial symptoms of congenital ectopia lentis (CEL). Carrying out studies from the perspective of ophthalmologists can promote the follow-up of lethal cardiovascular complications.

- This is the first study using qualitative research methods to discuss the compliance of CEL patients to cardiovascular follow-up.

- Ophthalmologists and medical anthropologists participated in the design and process of the study.

- The study is deficient in representativeness due to purposive sampling from one hospital. Research should be conducted using a wider sample size throughout the country in the future. Questionnaire also can be designed according to our results to expand the sample size and verify our findings.

- In order to obtain more comprehensive results, interviews of ophthalmologists to gauge their perspective should be conducted as well in future study.

life, they normally receive timely treatment. However, the development of cardiovascular complications is progressive and most will occur slowly with age and usually do not show noticeable symptoms before the age of $20 .{ }^{5}$ Patients will face the risk of sudden death once the symptoms appear. For patients at risk of developing cardiovascular lesions, regular monitoring is required. Life expectancy can be extended from 30 to 72 years through prophylactic treatment or surgery once cardiovascular abnormalities are found. ${ }^{67}$

Although regular monitoring, prophylactic medication and timely surgical intervention can significantly postpone the progression of disease and reduce the occurrence of cardiovascular accidents, there is still a large proportion of patients with CEL who have received treatment only in the later stages of the disease. ${ }^{8}$ During clinical practice, we have found that there hardly any patients proceed with regular follow-up visits with 
cardiovascular specialists. This phenomenon constitutes the core issue of this study: what factors hinder CEL patients' regular follow-up visits to cardiovascular specialists? Previous research has shown that the various clinical manifestations of CEL have increased the difficulty of diagnosis. ${ }^{3}$ Moreover, physicians, in addition to patients, lack awareness of the disease, which has affected physicians' diagnosis and reduced patients' compliance. ${ }^{9} 10$ Groth KA et al $^{11}$ compiled data on 412 patients with Marfan syndrome and found that 150 patients suffered from cardiovascular incident and 48 patients experienced sudden death. Among the 150 patients, 53 patients were diagnosed with Marfan syndrome after their first cardiovascular incident. According to the Chinese Rare Disease Report in $2018,{ }^{12}$ patients with rare genetic diseases such as Marfan syndrome have encountered many difficulties in seeking medical treatment. Lack of qualified specialists to provide information and guidelines, lack of adequate professional institutions and differences in physicians' and patients' perceptions of rare diseases may lead to the decline in patients' compliance.

Previous study on factors influencing CEL patients' follow-up visits were quantitative studies, which can successfully capture results, but not the detailed process of patient compliance. ${ }^{13}$ Patient compliance is a complex behaviour which is influenced by factors at multiple levels such as culture and psychological cognition. Thus, this study aims to give insight into CEL patients' careseeking behaviour and explore the factors affecting their follow-up visits by the qualitative methods.

\section{MATERIALS AND METHODS}

The study report is based on the consolidated criteria for reporting qualitative health research (COREQ). ${ }^{14}$

\section{Study design}

This research design was informed by grounded theory approaches. The semistructured interviews were conducted with patients suffering from CEL and their parents. The participants were recruited by using a purposive sampling frame in a large eye hospital in southern China, from 1 June 2017 to 1 January 2018. Inclusion criteria were having a clinical diagnosis of CEL, and exclusion criteria were having a lens dislocation caused by trauma or secondary to tumour and surgical operation. The preliminary interview guide was formed based on previous research and discussions with experienced ophthalmologists and medical anthropologists. The final guideline was finalised after pre-testing with five CEL patients.

\section{Data collection}

The interviews were conducted in a quiet examination room by an ophthalmologist and an undergraduate anthropology student. The conversations lasted about 30-60 min and the audio was recorded. The records were transcribed verbatim to ensure accuracy. Feedback was

\begin{tabular}{|c|c|}
\hline Basic information & Number $(n=35)$ n (\%) \\
\hline Male & $22(62.9 \%)$ \\
\hline Age, mean $( \pm S D)$, range & $11.4( \pm 8.3), 4-35$ \\
\hline First presentation in ophthalmology & $31(88.6 \%)$ \\
\hline Sporadic cases & $18(51.4 \%)$ \\
\hline Family history of SLD & $11(31.4 \%)$ \\
\hline Family history of CVC, SD & $6(17.2 \%), 3(9 \%)$ \\
\hline \multicolumn{2}{|l|}{ Education of the interviewees } \\
\hline Bachelor degree or above & $9(25.7 \%)$ \\
\hline High school & $5(14.3 \%)$ \\
\hline Junior high school or below & $21(60 \%)$ \\
\hline
\end{tabular}

CVC, cardiovascular complications; SD, sudden death; SLD, simple lens dislocation.

obtained from participants on the research findings. Data collection was closed when new themes did not appear in subsequent dialogues.

We interviewed 35 CEL patients and their parents. From the cohort of 35 patients, 31 patients had ophthalmology abnormalities as the initial symptom, 2 patients had cardiac abnormalities and 2 patients had skeletal abnormalities. Seventeen participants were able to provide a family history, and 11 of the 17 participants had a family history of simple lens dislocation. Six participants had a family history of cardiovascular complications and three families experienced sudden death. Among them, 30 participants were parents and 5 participants were patients themselves (the demographics information of participants is shown in table 1 ).

\section{Data analysis}

Audio-taped interviews were transcribed into written materials by two researchers ( $\mathrm{YZ}$ and $\mathrm{YC}$ ). After proofreading each other, the interviewees were asked to confirm the data to ensure more accurate. Then the written materials were imported into a software (Nvivo) for qualitative data management ( $\mathrm{YZ}$ and $\mathrm{YC}$ ). The thematic analysis and development of coding framework were performed by two researchers (JL and NG). First, JL and NG coded the transcripts line-by-line, respectively, and formed the open coding after discussion. To further develop axial coding and selective coding, a team discussion was organised every 2 weeks by all authors. Finally, codes were grouped into related categories and sorted into a draft framework of five main themes.

\section{Patient and public involvement}

Two patient advisers participated in the pilot study to help us refine the interview questions guide. Patients were not involved in the recruitment and conduct of the study. The information will be disseminated to the participants and public via the social platform. 


\section{RESULTS}

In our research, most of the interviewees said they never realised that CEL is a life-threatening disease. Neither physicians nor those in their social networks had informed them that CEL may endanger their life. In addition, shame on hereditary disease and busy with livelihood also hindered their follow-up. Thus, we categorised the data into five themes: insufficient awareness of CEL, shame on hereditary disease, lack of effective doctor-patient communication, lack of reliable information online and daily stressors.

\section{Insufficient awareness of CEL: no symptoms means no sickness}

Due to the lack of awareness of CEL, many parents fail to understand their children's health condition. They believed that the only treatment needed was for ocular symptoms, and a child who did not display discomfort was 'healthy'. Because of this, regular follow-ups were not prioritised unless further symptoms emerged.

I didn't know the disease could affect the whole body, I was just concerned about my daughter's poor vision. At that time, physicians said that there would be echocardiogram since they were worried about my daughter's heart problems. But I thought there couldn't be anything wrong with her heart because my daughter had been so healthy ever since birth. (NO 7)

Even in some cases, the disease was still not able to raise enough awareness even if a family member had experienced sudden death. In the absence of regular cardiovascular system monitoring, an appearance of clinical symptoms can cause serious consequences.

“'I didn't take my son to check his heart. Although my husband died young, I didn't link the two diseases together. I knew nothing about this disease at that time, and the doctor didn't mention anything, either. My son seemed healthy and I let it go. I didn't know the consequences would be so serious.' (NO 1, her husband suffered from sudden death, the son was diagnosed with CEL and showed cardiovascular symptoms. No regular follow-up had been completed until her husband's death.)

In our study, even though some physicians had given details of CEL to patients and their parents, there were still many people who believed that negative events would never happen to their families, a form of the psychological phenomenon of the 'optimism bias'.

I once had a suspicion that the disease was hereditary because my husband had problems in both his heart and his eyes, and underwent heart surgery before. I was worried that my child might also have a heart problem. He did an echocardiogram last year on the advice of a doctor, but the test result was all good. I think it should only happen by chance. The child is now in good health, there is no need to continue to check his heart. (NO 5, the husband experienced aortic surgery)

\section{Shame on hereditary disease}

In addition to counting on their luck, the traditional taboo for the disease is also an important factor in preventing regular follow-ups. As a traditional Chinese saying goes, 'family shame should not be made public'. Most CEL patients have a family history of the disease, and some patients even have multiple family members who are sick. They are embarrassed to talk about the disease in front of people, let alone seek medical advice.

I've had poor eyesight since childhood. I went to my optometrist but failed to get a pair of glasses. Meanwhile, my parents knew that the disease was inherited, but they were embarrassed, and did not want others to know or take me to see a physician. Now, I am blind to this disease, and never intended to learn about this. The only thing I know is that it's bad for my eyes. (NO 8 multiple family members with CEL, very embarrassed about disease, and not willing to talk about it)

In one case, having relatives experience sudden death or relatives that underwent aortic surgery caused interviewees to fear the disease. Consequently, one parent was reluctant to face the reality of his daughter's condition, which may have delayed the seeking of treatment.

I knew about Marfan syndrome long before. Although my child is still quite healthy except for her poor eyesight, I still worry about her heart. However, I was so afraid of knowing whether there was a problem with her heart so I never dared to take her to see physicians. In the past, my sister had gone into cardiac arrest several times during her heart surgery, it was really horrible. I wondered if there was any medication to prevent my daughter from needing surgery. (NO 20, sudden death or aortic surgery in multiple family members)

\section{Lack of effective doctor-patient communication}

CEL is a rare genetic disease with complicated clinical manifestations, and many ophthalmologists are unfamiliar with it. When seeing patients, ophthalmologists tend to focus on patients' ocular symptoms but fail to eliminate possible systemic complications.

Some patients complained: I discovered that my son had pectus carinatum as long as he had his eyes problems when he was an infant. We thought that his eyesight might improve as he grew up, so we didn't take him to see physicians. Then, the local doctor said my son had CEL, and surgery must be performed as soon as possible and yet no other examinations had been ordered. We are uneducated and we do whatever the physicians tell us. (NO 2, daughter also has CEL and visited county hospital) 
When I took my daughter to the county hospital, the doctor there only suggested that my daughter should fill an optometry prescription but did not further explain possible complications to us. (NO 22, interviewee himself and his two daughters diagnosed with CEL)

Situations like the above one was also experienced by other interviewees of our study. More than half of the participants expressed that physicians did not recommend a full-body examination during their first consultation, and most of their physicians were from county hospitals. This suggests that rare diseases do not get much attention in primary hospitals. Physicians tend to focus on their own field and may neglect a patients' general condition. As for cases in large public hospitals, even though most physicians are familiar with CEL and its possible complications, they may still fail to thoroughly explain the condition to patients. Therefore, patients are not aware of the risk of cardiovascular complications. This affects their compliance and can lead to serious consequences.

This disease is very rare. Doctors asked us to check my child's heart to find out if there were any problems. He (the doctor) didn't tell me specifically why he ordered the examination. At that time, I hadn't heard of Marfan syndrome at all, so I just got my child a pair of glasses and didn't take it to be serious. (NO 15)

It is a common occurrence that the unsuccessful delivery of information from a physician can lead to a patient's deteriorating condition. Some patients may not return to hospitals until severe cardiovascular complications occur, thereby missing the best opportunity for treatment.

I took my son to the hospital to check his eyes because he couldn't see clearly, and the doctor suspected that it was Marfan syndrome and was prepared to perform eye surgery for him. However, the surgery was postponed since the ECG indicated some heart problems, so I could only get him glasses instead. The doctor didn't specifically inform us of how serious the condition was. (NO 1)

Sadly, in some cases, situations can become even more extreme. Physicians fail to convey possible serious complications of the disease to patients during consultation, resulting in tremendous tragedy.

My brother passed out and died suddenly after 2 months after having eye surgery. I went back to the hospital to ask for an answer after his sudden death, and the doctor said that his artery may have ruptured. I was furious knowing that my brother may have survived if we knew the condition earlier and proceeded with the heart surgery. Why didn't they tell us that there were problems with my brother's heart? (NO21, mother experienced sudden death, and younger brother experienced sudden death 1 month after CEL surgery)

\section{Lack of reliable information online}

Many patients use other platforms including the search engine or social media to learn about their condition because they cannot obtain satisfactory information from physicians. However, information online is unreliable and can lack accuracy. Patients searching for information online are often do not obtain reliable information.

'I started to search for information after having heart issues, but what I acquired was limited. I also joined some discussion groups, but there are too many commercials. I chose to ignore those. Those are not reliable.' When asked whether he believed the information, he said, 'I can only learn about information here, I don't know if it is reliable. I am sure about the information about the cardiovascular system and the eyes. I'm not sure about other information since I don't have those symptoms. There was a patient who had the same disease as me. He was very tall. His eyes were fine but had problems with his aorta.' (NO 1)

There is a lot of information about CEL online, but none of those sources are comprehensive. Disaccredited hospitals publish fake advertisements in order to draw patients' attention. Even if patients can identify the authenticity of such hospitals, they may be unable to seek medical consultation.

I've searched the Internet for the pathogenesis and complications of the disease, and I know it's harmful to my life. I also know that there is no effective surgery or medications to cure me. Nowadays there are many links to hospitals on the internet, and I think they are not credible, they look fraudulent. Local hospitals can't cure me, and I don't know where to go. To be honest, I want to see doctors outside of our town, but I don't know how to take the first steps. (NO 35, multiple members in family have CEL)

There are also some parents who searched for additional sources of information because they were worried about their child's condition. They searched for information online and consulted with physicians repeatedly before finally determining the therapeutic plan for their children, and successfully avoided a tragic outcome.

The doctor did not mention that there would be other problems. We searched Baidu for information on CEL and knew it could be Marfan syndrome. We ordered an echocardiography and found out that there was minor extension in the aortic sinus. We asked the doctor to prescribe some oral medication. Genetic tests and bone examinations were also performed. (NO 28, both parents have bachelor degree)

The information online can be convoluted and unreliable, which confused many patients. Although some parents obtained authentic information about the disease by themselves, most of them had delayed medical treatment due to lack of information. 


\section{Daily stressors}

Many parents tend to underestimate the serious complications later in life due to the satisfactory treatment outcome of ocular symptoms during their children's childhood. In our study, making a living remains a priority for parents and follow-ups are postponed unless severe complications occur.

My husband is always on business trips, and I have to go to work. I am tied up at work. It is very difficult to have regular follow-ups with doctors. Each time we see the doctor we only have our child have his eyes examined. We don't know much about the disease and have not learned about it. We mainly listen to what the doctors say. (NO 6, no family history)

The doctor said that our child recovered well after surgery for lens dislocation, meanwhile there was no abnormality in his heart. I was very busy at work and our child had to go to school, so I thought it wasn't necessary to have a follow-up. We would see a doctor if there was anything wrong. (NO 32, no family history)

A large number of patients have a family history of CEL, poor eyesight and other severe complications that negatively impact the patients' life that can lead to poor education and income. Moreover, many families are under enormous economic stress when one or more members in the family get diagnosed with CEL.

My eyesight has always been poor. I wasn't able to see a doctor because of financial problems. I had surgery when I had some money, but it cost me all of my savings. (NO 8, multiple CEL patients in family)

My dad took me to the county hospital when I was a child. I had the opportunity to have surgery, but it never happened since it would have cost us 20,000 RMB which our family couldn't afford. My mother had heart surgery. Actually, there were problems with her heart twoyears leading up to her surgery, but we still had to postpone the surgery until our financial condition improved. (NO 19, CEL patient with aortic ectasis whose mother underwent heart surgery and son has CEL)

\section{DISCUSSION}

Cardiovascular complications are extremely harmful to CEL patients, and in some serious cases, patients may even experience sudden death. Previous studies have found that regular monitoring of the cardiovascular system is vital to CEL patients, and life expectancy can be significantly improved by oral losartan or cardiovascular surgery. ${ }^{15}$ However, most of the patients who came to the hospital seeking medical consultation did not have regular cardiovascular examinations. Our study indicates that most of the interviewees had never realised the serious complications that CEL may cause. According to their understanding of the disease, they thought that an absence of discomfort equated to an absence of the disease. This is the primary reason that patients and parents did not seek regular follow-ups with cardiovascular specialists. There are five main reasons for this lack of risk perception: insufficient awareness of CEL, shame on hereditary disease, lack of effective doctor-patient communication, lack of reliable information online and daily stressors.

First, in our study, more than half of the participants reported that the ophthalmologist did not inform them of possible systemic complications, nor did they recommend general examinations. These physicians, mainly from county hospitals, simply focussed on treating CEL, and some even misdiagnosed CEL as another disease. According to the Chinese health and family planning statistical yearbook in $2017,{ }^{16}$ only $28.5 \%$ of medical practitioners in China have bachelor degree or above, and the others have not received systematic and normative medical education. Physicians in primary hospitals generally have lower academic qualifications and less chance of encountering rare diseases, all of which leads to their insufficient awareness of rare diseases. Sixty-five per cent of rare disease patients were once misdiagnosed, and less than a quarter of them were able to obtain a definitive diagnosis in the first consultation. ${ }^{12}$ In consideration of this situation, medical institutions should organise regular training projects for medical practitioners that focus on rare diseases that may endanger patients' lives such as CEL. We believe that training projects like this could raise physician awareness of severe, rare diseases and avoid misdiagnosis or delayed treatment.

Second, although physicians in top-level hospitals are familiar with CEL and will recommend systemic examinations, they are still unable to explain in detail the condition to the patient. As such, many patients are not clear about the severity of the disease and their compliance remain poor. Patients in the most developed countries have sufficient time to communicate with physicians since they make appointments and see physicians in private clinics. However, in China, according to 2012 data, $90 \%$ of patients see physicians in crowded public hospitals. ${ }^{17}$ In contrast to the scenario in developed countries, patients in China tend to visit top-level hospitals due to the uneven distribution of medical resources. Unfortunately, even physicians in top hospitals are expected to see a large number of patients, making it difficult to communicate with patients thoroughly. Even if physicians are willing to explain in detail the patient's condition, treatment options or potential risks, patients may not understand the medical jargon considering the gap between physician and patient knowledge. The process of the consultation is intended to be reciprocal, which means physicians should offer accurate medical advice in accordance with patients' level of comprehension. ${ }^{18}$ Studies show that China's medical education concentrates on clinical or scientific capabilities instead of doctor-patient communication. ${ }^{19}$ Indeed, the importance of doctor-patient communication does appear in 
textbooks, but field education and assessment does not yet exist. The importance of doctor-patient communication cannot be stressed enough, therefore, training and assessment by the health department should be made a priority.

Third, patients tend to acquire information through other channels if they cannot receive adequate information from physicians. Among those channels, the internet seems to be the primary source. In our study, many patients complained that online information usually contained a large amount of advertisements, and questioned the authenticity of the information. However, this is not to say that all secondary sources of medical information are not beneficial. In 2010, the first online public welfare organisation 'Porcelain-Doll Rare Diseases Care Centre' was established in China. ${ }^{20}$ Since then, more and more organisations have been founded to help rare diseases patients. Likewise, organisations aiming at Marfan syndrome were established. These organisations have made efforts to promote doctor-patient communication and propagate knowledge about recent research about the disease. In addition, financial aid for patients with difficulties was offered. However, compared with other similar financial aid institutions in other countries, ${ }^{21}{ }^{22}$ those in China remain rudimentary. These organisations struggle with receiving insufficient support from government administrations. Consequently, these organisations have limited influence and reach to benefit patients throughout the country. We propose that the health department, physicians and patients should make a joint effort to build an effective information-sharing platform to promote reliable information on the diagnosis and treatment of CEL.

At the same time, patients with CEL are incurring considerable costs throughout the process of the treatment. Each step, starting ophthalmology treatment during childhood (optometry or eye surgery), to annual examinations and oral medications for prophylaxis, and ending with cardiovascular surgery, is quite expensive. Moreover, the financial burden may become heavier if more than one member in the family has CEL. Meanwhile, poor eyesight and other severe complications will have a negative impact on a patient's life and capacity to earn a steady income. Achelrod $e t a l^{23}$ conducted statistical analysis on the annual medical expenditure of 892 patients with Marfan syndrome. They discovered that their expenditure increased by 2496 euros (approximately equal to $20000 \mathrm{RMB}$ ) compared with healthy individuals. In China, the coverage of healthcare for rare diseases is so low that almost $70 \%$ of the expenditure comes from patients themselves. Among them, the cost for rural-dwelling patients is 1.42 times that of their personal income, which is $78.1 \%$ of their annual family income. ${ }^{12}$ Policy makers should improve the healthcare policy for rare, genetic diseases, and set up certain medical assistance programme along with welfare organisations to relieve the economic pressure on patients and improve their compliance.

\section{CONCLUSION}

We have discovered five factors that affect CEL patient or parents from attending a cardiovascular follow-up: insufficient awareness of CEL, shame on hereditary disease, lack of effective doctor-patient communication, lack of reliable information online and daily stressors. These factors can eventually lead to a result that is far from ideal: parents who are unaware of the potential serious consequences of CEL and believing that children who have no symptom are healthy. In order to promote the status of diagnosis, treatment and patients' compliance for CEL, certain methods should be carried out. The health department and physicians can collaborate to raise physicians' awareness through organising training projects of rare diseases such as CEL. They can also collaborate to promote the importance of patient-physician communication through reforming the education model. Third, they can aid in broadening patients' access to medical knowledge through building a reliable information platform. Lastly, the government could partake in relieving the economic pressure on patients through improving the healthcare policy for rare genetic diseases.

Acknowledgements We would like to acknowledge the patients and family members who participated in this study. We also thank patient advisers who helped us to refine the interview question guide.

Contributors $\mathrm{JL}$ and NG contributed equally to this work, and they are joint first authors. JL and NG were involved in question development, data collection and analysis and manuscript preparation. QC participated in data analysis and manuscript preparation. $Y Z$ and $Y C$ were involved in the interviews of the patients, data collection and manuscript preparation. GJ, CAY, JY and YW were involved in literature review and manuscript preparation. DZ was involved in question development and manuscript preparation. All authors read and approved the final manuscript

Funding This study was supported by National Natural Science Foundation of China, Grant/Award Number: 81873673.

Competing interests None declared.

Patient consent for publication Parental/guardian consent obtained.

Provenance and peer review Not commissioned; externally peer reviewed.

Data availability statement Data are available upon reasonable request.

Open access This is an open access article distributed in accordance with the Creative Commons Attribution Non Commercial (CC BY-NC 4.0) license, which permits others to distribute, remix, adapt, build upon this work non-commercially, and license their derivative works on different terms, provided the original work is properly cited, appropriate credit is given, any changes made indicated, and the use is non-commercial. See: http://creativecommons.org/licenses/by-nc/4.0/.

ORCID iD

Jianqiang Lin http://orcid.org/0000-0001-5651-1932

\section{REFERENCES}

1 Fuchs J, Rosenberg T. Congenital ectopia lentis. A Danish national survey. Acta Ophthalmol Scand 1998;76:20-6.

2 Sadiq MA, Vanderveen D. Genetics of ectopia lentis. Semin Ophthalmol 2013;28:313-20.

3 Ho NCY, Tran JR, Bektas A. Marfan's syndrome. Lancet 2005;366:1978-81.

4 Tinkle BT, Saal HM, Committee on genetics. Health supervision for children with Marfan syndrome. Pediatrics 2013;132:e1059-72.

5 Loeys BL, Dietz HC, Braverman AC, et al. The revised Ghent nosology for the Marfan syndrome. J Med Genet 2010;47:476-85.

6 Hoffmann BA, Rybczynski M, Rostock T, et al. Prospective risk stratification of sudden cardiac death in Marfan's syndrome. Int $J$ Cardiol 2013;167:2539-45. 
7 Miyahara S, Okita Y. Overview of current surgical strategies for aortic disease in patients with Marfan syndrome. Surg Today 2016;46:1006-18.

8 Blankart CR, Milstein R, Rybczynski M, et al. Economic and care considerations of Marfan syndrome. Expert Rev Pharmacoecon Outcomes Res 2016:16:591-8.

9 von Kodolitsch Y, De Backer J, Schüler H, et al. Perspectives on the revised Ghent criteria for the diagnosis of Marfan syndrome. Appl Clin Genet 2015;8:137-55.

10 Kainulainen K, Pulkkinen L, Savolainen A, et al. Location on chromosome 15 of the gene defect causing Marfan syndrome. $N$ Engl J Med 1990;323:935-9.

11 Groth KA, Stochholm K, Hove H, et al. Aortic events in a nationwide Marfan syndrome cohort. Clin Res Cardiol 2017;106:105-12.

12 China Development Brief. China rare disease survey report in 2018. Available: http://www.chinadevelopmentbrief.org.cn/news-21008. html [Accessed 28 Feb 2018].

13 Malterud K. Qualitative research: Standards, challenges, and guidelines. Lancet 2001;358:483-8.

14 Tong A, Sainsbury P, Craig J. Consolidated criteria for reporting qualitative research (COREQ): a 32-item checklist for interviews and focus groups. Int J Qual Health Care 2007:19:349-57.
15 Radke RM, Baumgartner H. Diagnosis and treatment of Marfan syndrome: an update. Heart 2014;100:1382-91.

16 Chinese health and family planning statistical Yearbook in 2017. Available: http://www.yearbookchina.com/navibooklistN2017120225-1.html [Accessed 1 Sep 2017].

17 Bao Y, Fan G, Zou D, et al. Patient experience with outpatient encounters at public hospitals in Shanghai: examining different aspects of physician services and implications of overcrowding. PLoS One 2017;12:e0171684.

18 Kluge E-HW, Competence KEH. Competence, capacity, and informed consent: beyond the cognitive-competence model. Can J Aging 2005;24:295-304.

19 Song P, Tang W. Emphasizing humanities in medical education: promoting the integration of medical scientific spirit and medical humanistic spirit. Biosci Trends 2017:11:128-33.

20 Wang J-B, Guo JJ, Yang L, et al. Rare diseases and legislation in China. Lancet 2010;375:708-9.

21 Marfan World. Available: http://www.marfanworld.org/

22 The Marfan Foundation. Available: http://www.marfan.org/about-us/ about

23 Achelrod D, Blankart CR, Linder R, et al. The economic impact of Marfan syndrome: a non-experimental, retrospective, populationbased matched cohort study. Orphanet J Rare Dis 2014;9:90. 